\title{
Journal of Cardiovascular Magnetic

\section{Management of the pregnant patient with complex congenital heart disease or aortopathy with cardiovascular magnetic resonance}

\author{
Marshall W Winner III*, Subha Raman and Stephen Cook
}

Address: The Ohio State University, Columbus, OH, USA

* Corresponding author

from I th $^{\text {th }}$ Annual SCMR Scientific Sessions

Los Angeles, CA, USA. I-3 February 2008

Published: 22 October 2008

Journal of Cardiovascular Magnetic Resonance 2008, I0(Suppl I):A197 doi:I0.I I86/I532-429X-I0-SI-A197

This abstract is available from: http://jcmr-online.com/content/I0/SI/AI 97

(c) 2008 Winner et al; licensee BioMed Central Ltd.

\section{Background}

Increasing numbers of women with congenital heart disease (CHD) are now reaching childbearing age. Not uncommonly, women often present for clinical evaluation in the gravid state after years of little or no follow-up. Due to their complex anatomy, it is often difficult to obtain the anatomic and functional information necessary to appropriately manage these patients throughout their pregnancy. We investigated the ability of cardiac magnetic resonance (CMR) to provide anatomic and functional data that further assisted in the management of this challenging patient population.

\section{Methods}

We performed a retrospective study of pregnant women with complex CHD or aortopathies who underwent CMR between June 2005 and April 2007. We assessed the ability of CMR to visualize anatomy and ventricular function. If transthoracic echocardiogram (TTE) was performed, additional information gained from CMR was noted (Figure 1). We also determined if CMR affected the management of each patient throughout pregnancy.

\section{Results}

Fourteen patients with complex CHD or aortopathy underwent CMR during pregnancy. Eleven patients (79\%) presented for the first time to the Adult CHD clinic at an average of 22.5 weeks gestation. There were 3 patients with bicuspid aortic valve and dilated ascending aorta, 2 each with coarctation of the aorta (CoA) and tetralogy of
Fallot (TOF), and 1 each with Marfan's syndrome, Ebstein's anomaly, double chamber right ventricle (DCRV), congenitally corrected transposition of the great arteries (cc-TGA), d-TGA status-post Senning repair, restrictive cardiomyopathy and a mitral valve mass. Thirteen patients (93\%) also underwent a TTE during their pregnancy.

\section{Imaging evaluation}

CMR provided a thorough assessment of cardiac anatomy and ventricular volumes and function in all fourteen patients. In all cases of aortopathy, CMR was able to fully visualize the aorta and aortic valve. Aortopathy patients' aortic dimensions were measured throughout; in one case, the ascending aorta measured $4.8 \mathrm{~cm}$ though TTE reported only a maximum root dimension of $2.8 \mathrm{~cm}$. In both patients with TOF, including one with a history of a palliative Waterston shunt, CMR was able to define right and left ventricular function and aortic and pulmonary artery anatomy. CMR quantified right and left ventricular function in patients with both forms of TGA, DCRV, and Ebstein's anomaly. The mean right ventricular end-diastolic volume, end-systolic volume and ejection fraction was $126 \mathrm{ml}, 70 \mathrm{ml}$, and 44\% respectively. Comparable values for the left ventricle were $97 \mathrm{ml}, 41 \mathrm{ml}$, and 59\%, respectively.

CMR provided additional anatomic information beyond that provided by TTE in 7 patients. TTE suggested CoA in 2 patients. Further imaging with CMR concluded a cervi- 


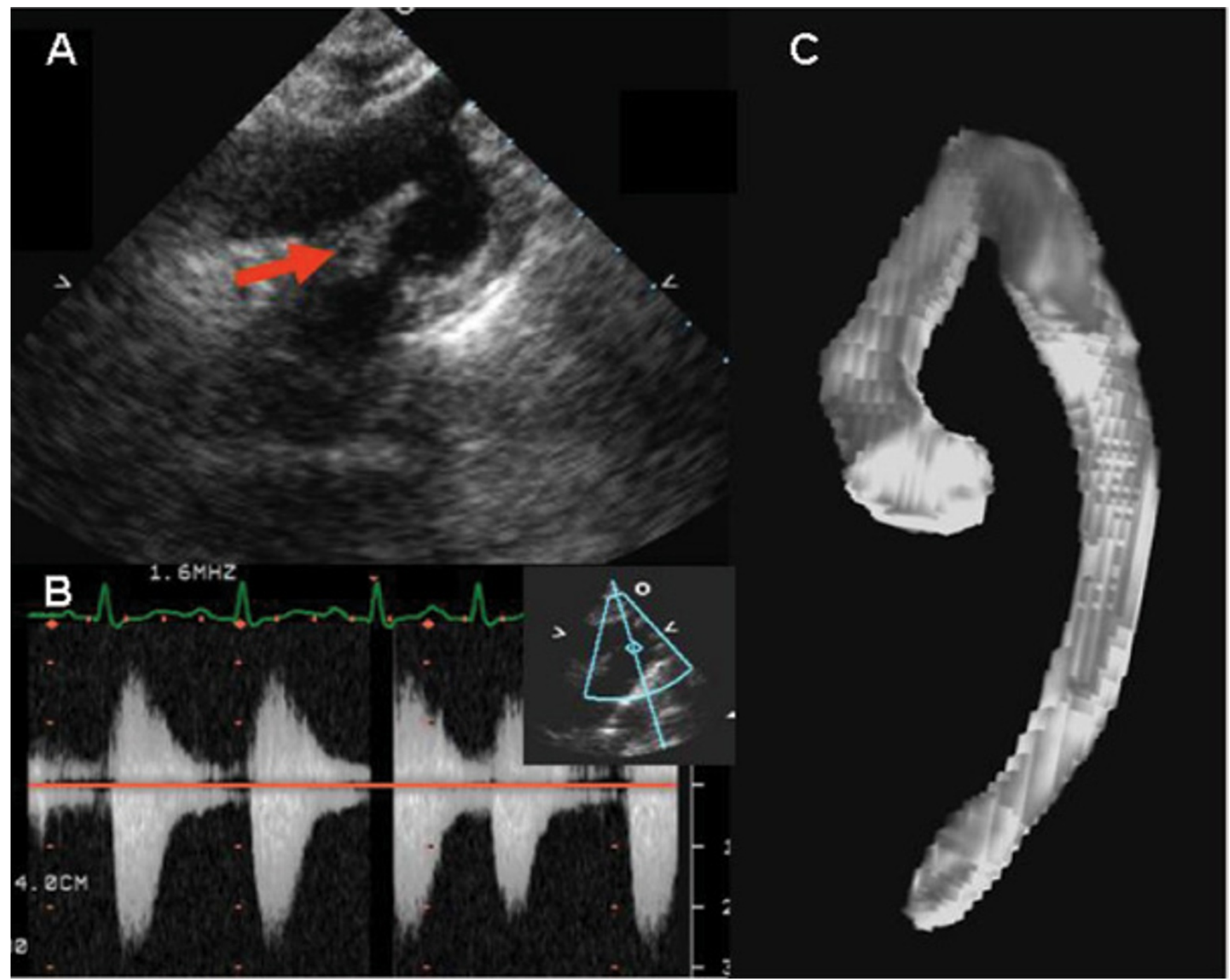

\section{Figure I}

26 week pregnant patient in whom coarctation of the aorta was suspected following TTE. CMR confirmed the diagnosis of cervial arch without coarctation. (A) TTE of aortic arch demonstrating a possible posterior shelf (arrow). (b) Continuous Doppler tracing of the aortic arch estimates a peak gradient of $25 \mathrm{mmHg}$. (C) Volume rendered non-contrast CMR image of entire thoracic aorta.

cal arch in one patient and tortuous aorta without stenosis in the second, confirming the absence of CoA in both patients. CMR provided accurate information regarding the systemic and pulmonary venous baffles in the patient with d-TGA.

\section{Clinical decision making}

In 7 (50\%) of cases, CMR results affected patient management. One patient with Marfan's was delivered urgently, and two patients underwent Cesarean-section due to aortic aneurysms. Two patients with CoA and one patient with a dilated ascending aorta were managed conservatively undergoing vaginal delivery after CMR determined absence of significant aortic disease. Finally, in the patient with d-TGA, CMR defined previously unknown cardiac anatomy and function, facilitating appropriate medical management.

\section{Conclusion}

CMR safely assesses cardiovascular anatomy and function in pregnant patients with CHD and aortopathy. In 50\% of the cases evaluated, CMR provided additional information beyond that of TTE for the physicians caring for these patients and ultimately played an essential role in the clinical management of this complex group of patients. CMR should be utilized when it may impact upon optimal management of the complex CHD patient during pregnancy. 